OPEN ACCESS

Edited by:

Venkatesh Sundararajan,

the State University of New Jersey,

United States

Reviewed by:

Ashutosh Pandey,

Rutgers Biomedical and Health

Sciences, United States

Tamer M. A. Mohamed,

University of Louisville, United States

*Correspondence:

Hangyuan Guo

guohangyuan@zju.edu.cn

tThese authors have contributed equally to this work

Specialty section:

This article was submitted to Cardiovascular and Smooth Muscle Pharmacology,

a section of the journal

Frontiers in Pharmacology

Received: 26 April 2021 Accepted: 04 June 2021

Published: 24 June 2021

Citation:

Lin H, Guan L, Meng L, Uzui $H$ and Guo H (2021) SGLT1 Knockdown Attenuates Cardiac Fibroblast Activation in Diabetic Cardiac Fibrosis.

Front. Pharmacol. 12:700366.

doi: 10.3389/fphar.2021.700366

\section{SGLT1 Knockdown Attenuates Cardiac Fibroblast Activation in Diabetic Cardiac Fibrosis}

\author{
Hui Lin ${ }^{1 \dagger}$, Le Guan ${ }^{2 \dagger}$, Liping Meng ${ }^{1}$, Hiroyasu Uzui ${ }^{3}$ and Hangyuan Guo ${ }^{4 *}$ \\ ${ }^{1}$ Department of Cardiology, Shaoxing People's Hospital, Shaoxing Hospital, Zhejiang University School of Medicine, Shaoxing, \\ China, ${ }^{2}$ Department of Radiology, Shaoxing People's Hospital, Shaoxing Hospital, Zhejiang University School of Medicine, \\ Shaoxing, China, ${ }^{3}$ Department of Cardiovascular Medicine, Faculty of Medical Sciences, University of Fukui, Fukui, Japan, \\ ${ }^{4}$ College of Medicine, Shaoxing University, Shaoxing, China
}

Background: Cardiac fibroblast (CF) activation is a hallmark feature of cardiac fibrosis in diabetic cardiomyopathy (DCM). Inhibition of the sodium-dependent glucose transporter 1 (SGLT1) attenuates cardiomyocyte apoptosis and delays the development of DCM. However, the role of SGLT1 in CF activation remains unclear.

Methods: A rat model of DCM was established and treated with si-SGLT1 to examine cardiac fibrosis. In addition, in vitro experiments were conducted to verify the regulatory role of SGLT1 in proliferation and collagen secretion in high-glucose- (HG-) treated CFs.

Results: SGLT1 was found to be upregulated in diabetic cardiac tissues and HG-induced CFs. HG stimulation resulted in increased proliferation and migration, increased the expression of transforming growth factor- $\beta 1$ and collagen I and collagen III, and increased phosphorylation of p38 mitogen-activated protein kinase and extracellular signal-regulated kinase (ERK) 1/2. These trends in HG-treated CFs were significantly reversed by si-SGLT1. Moreover, the overexpression of SGLT1 promoted CF proliferation and collagen synthesis and increased phosphorylation of p38 mitogen-activated protein kinase and ERK1/2. SGLT1 silencing significantly alleviated cardiac fibrosis, but had no effect on cardiac hypertrophy in diabetic hearts.

Conclusion: These findings provide new information on the role of SGLT1 in CF activation, suggesting a novel therapeutic strategy for the treatment of DCM fibrosis.

Keywords: cardiac fibroblasts, sodium-glucose cotransporter, high glucose, diabetic cardiomyopathy, mitogenactivated protein kinase

\section{INTRODUCTION}

Diabetic cardiomyopathy (DCM) is a myocardial disease that is specific to patients with diabetes and is independent of various types of heart diseases, including hypertension, coronary, and valvular (Bugger and Abel, 2014; Seferovic and Paulus, 2015). Cardiac fibrosis caused by abnormal glucose metabolism and microangiopathy are the main pathological features of DCM, leading to impairment

Abbreviations: DCM, diabetic cardiomyopathy; CFs, cardiac fibroblasts; SGLT1, sodium-glucose cotransporter; FBG, fasting blood glucose; IPGTTs, intraperitoneal glucose tolerance tests; HG, high glucose; MAPK, mitogen-activated protein kinase; ERK, extracellular signal-regulated kinase; TGF- $\beta 1$, transforming growth factor- $\beta 1$. 
of cardiac function and eventual progression to heart failure (Wang et al., 2021). The activation of cardiac fibroblasts (CFs) and degeneration of cardiomyocytes provide the biological basis for cardiac remodeling and the pathophysiological basis of DCM formation (Zhang et al., 2020). CFs switched from a resting type to an activated type increasing their proliferation and migration capacity and began to secrete large amounts of extracellular matrix, causing fibrosis of the heart (Frangogiannis, 2021). The development of fibrosis-targeting therapies for patients with DCM will help to further understand the functional pluralism of $\mathrm{CFs}$ and dissect the molecular basis for fibrotic remodeling.

Sodium-glucose cotransporter (SGLT) belongs to the solute carrier five gene family, which transports glucose against a concentration gradient in an energy-consuming manner and plays an important role in the active transport of glucose (Wood and Trayhurn, 2003; Sano et al., 2020). Sodium-glucose cotransporter 1 (SGLT1) is expressed in various human tissues and organs, including the intestine, lung, heart, skeletal muscle, and kidney (Gyimesi et al., 2020). SGLT1 is essential for the quick absorption of glucose and galactose in the intestine, and increases in SGLT1 protein expression cause interstitial fibrosis and cardiac remodeling in mice (Ramratnam et al., 2014). SGLT1 expression is also elevated in hypertrophic cardiomyopathy, ischemic cardiomyopathy, and DCM in humans (Song et al., 2016). Selective inhibition of SGLT1 expression has a protective effect against myocardial-infarctioninduced ischemic cardiomyopathy (Sawa et al., 2020). In addition, Hirose et al. (2018) demonstrated that SGLT1 knockout effectively alleviated pressure-overload-induced cardiomyopathy, suggesting that SGLT1 inhibitors have an active effect on hypertrophic cardiomyopathy. More importantly, our previous study found that SGLT1 inhibition could attenuate apoptosis and relieve myocardial fibrosis, thus suppressing DCM development by regulating the JNK/p38 signaling pathway (Lin et al., 2021). However, in the abovementioned study, we only investigated the role of SGLT1 in cardiomyocytes and rat $\mathrm{H} 9 \mathrm{C} 2$ cells. It would be more appropriate to study the role of SGLT1 in the activation of CFs during the development of DCM.

Our previous study found that high-glucose (HG) levels promote SGLT1 and matrix metalloproteinase 2 expression in CFs (Meng et al., 2018), but whether HG levels promote cardiac fibrosis by inducing CF activation and whether SGLT1 is involved in HG-induced CF activation have not been reported. Thus, arrays of experiments were performed in this study to determine the role of SGLT1 in CF activation during DCM. Moreover, we tried to characterize the role of the p38 and ERK1/2 signaling pathways in the regulatory mechanism of SGLT1 expression for CF activation.

\section{MATERIALS AND METHODS}

\section{Ethics Statement}

All animal procedures were conducted in accordance with the National Institutes of Health Guide for the Care and Use of
Laboratory Animals and were approved by the Medicine Animal Welfare Committee of Shaoxing People's Hospital.

\section{Culture of Rat Primary CFs}

Primary rat CFs were isolated from the ventricles of neonatal male Sprague Dawley rats (2-3 days old) using enzyme digestion. The tissue was cut into $1 \mathrm{~mm}$ cube pieces and digested with trypsin/EDTA (Gibco, NY, United States) and collagenase II (Sigma, United States) at $37^{\circ} \mathrm{C}$. The mixture (collagenase and trypsin, 100: 1) was placed in a shaker at $37^{\circ} \mathrm{C}$ for $20 \mathrm{~min}$, and the supernatant was collected and combined with DMEM containing $10 \%$ FBS, and this process was repeated until the tissue was fully digested. Cardiomyocytes were separated from CFs using centrifugation at a low speed $(300 \mathrm{~g})$, and the supernatant containing CFs was collected. The isolated CFs were cultured in DMEM supplemented with $10 \%$ FBS and $1 \%$ penicillin-streptomycin at $37^{\circ} \mathrm{C}$ in a humidified incubator with $5 \% \mathrm{CO}_{2}$. Cells were divided into four groups: 1) a control group, in which CFs were incubated with DMEM containing $5.5 \mathrm{mmol} / \mathrm{L}$ normal glucose for $48 \mathrm{~h}$; 2) an HG group, in which CFs were incubated with DMEM containing $33 \mathrm{mmol} / \mathrm{L}$ glucose (HG) for $48 \mathrm{~h} ; 3)$ an $\mathrm{HG}+$ si-NC group, in which CFs were transfected with si-NC and cultured under HG conditions for $48 \mathrm{~h}$; and 4 ) an HG + si-SGLT1 group, in which CFs were transfected with si-SGLT1 and cultured under $\mathrm{HG}$ conditions for $48 \mathrm{~h}$.

\section{Small Interference RNA Transfection}

To knockdown the expression of SGLT1 in CFs, small interfering RNAs against the SGLT1 gene (si-SGLT1) and the siRNA negative control (si-NC) were synthesized at Guangzhou RiboBio Co., Ltd. Briefly, CFs grown to $70-80 \%$ confluence were incubated with Lipofectamine 3,000 transfection reagent (Invitrogen, Waltham, MA, United States) loaded with siRNAs for $48 \mathrm{~h}$. Transfection efficiency was evaluated using RT-qPCR analysis.

\section{Cell Counting Kit-8 Assay}

CFs were cultured in 96 -well plates at a density of $1 \times 10^{4}$ cells/ well. A $10 \mu \mathrm{l}$ aliquot of the Cell Counting Kit-8 (MCE, Shanghai, China) solution was added to each well, and the plates were incubated at $37^{\circ} \mathrm{C}$ for $1 \mathrm{~h}$. Absorbance was measured using a microplate reader (Molecular Devices, CA) at $450 \mathrm{~nm}$.

\section{Wound Scratch Assay}

Cells were grown to $90 \%$ confluence in 6 -well plates in DMEM supplemented with $10 \%$ FBS, and the medium was replaced with serum-free DMEM to starve cells for $24 \mathrm{~h}$. Wounds were made with a sterile $200 \mu$ pipette tip by drawing a line through the plated cells perpendicular to the abovementioned line. CFs were transfected with si-NC or si-SGLT1 and exposed to HG conditions for $24 \mathrm{~h}$. Images were acquired using a Leica microscope (DM 2000, Leica, Wetzlar, Germany). 


\section{Western Blotting}

Protein samples from CFs and cardiac tissues were extracted using the RIPA buffer, and equal amounts of proteins from each group were separated by $12 \%$ sodium dodecyl sulfatepolyacrylamide gel electrophoresis. Then, the separated proteins were transferred to polyvinylidene fluoride membranes and blocked using 5\% nonfat milk for $1 \mathrm{~h}$ at room temperature. Subsequently, the membranes were incubated with primary antibodies at $4^{\circ} \mathrm{C}$ overnight, followed by incubation with horseradish peroxidase- (HRP-) conjugated goat anti-rabbit secondary antibody (1:5,000, Santa Cruz) for $1 \mathrm{~h}$ at room temperature. The following primary antibodies were purchased from Abcam and used at a 1:1,000 dilution: $\beta$-actin (ab5694), SGLT1 (ab14686), collagen I (ab34710), and collagen III (ab6310). The following antibodies were purchased from Cell Signaling Technology and used at a 1:1,000 dilution: ERK1/2 (cat. 4695), phospho-ERK1/2 (cat. 4376), p38 (cat. 8690), and phospho-p38 (cat. 4511).

\section{RNA Analysis}

TRIzol reagent (Invitrogen, Carlsbad, CA, United States) was used to extract RNA from cardiac tissues, and cDNA was synthesized using a PrimeScript Reverse Transcription Reagent Kit (Takara, Otsu, Japan). RT-PCR was performed using a SYBR Premix Ex Taq Kit (Takara). The following primer sequences were used: SGLT1 forward, 5'-GGACAGTAGCACCTTGAGC$3^{\prime}$; reverse, $5^{\prime}$-CCAACAGTCCCACGATTAG- $3^{\prime} ; \quad \beta$-actin forward, $5^{\prime}$-CCAGATCATGTTTGAGACCT-3'; and reverse, $5^{\prime}$-TCTCTTGCTCGAAGTCTAGG-3' ${ }^{\prime}$. All reactions were performed in triplicate.

\section{Animal Experiments}

Sprague Dawley rats were obtained from the Nanjing Biomedical Research Institute of Nanjing University (China). A total of 24 6week-old male SD rats were assigned to four groups (control, STZ, si-NC, and si-SGLT1 groups) using the random number method, with six rats in each group. A 12-h light-dark cycle was used, and the rats were provided ad libitum access to food and water. After acclimation for 1 week, rats in the diabetes groups were fed a highfat diet (fat provided 60\% of total calories, Research Diet D12492) for 4 weeks and then intraperitoneally injected with $60 \mathrm{mg} / \mathrm{kg} \mathrm{STZ}$ (Sigma) dissolved in a citrate buffer ( $\mathrm{pH} 4.5)$, whereas the control group received normal chow. We performed intraperitoneal glucose tolerance tests (IPGTTs) to identify the insulin-resistant rats, and fasting blood glucose (FBG) levels were measured seven days after injection. Body weights were recorded. Successful induction of diabetes was defined by an FBG value higher than $16.7 \mathrm{mmol}$ (Feng et al., 2019). After successful establishment of the rat model with DCM, the rats in the si-NC and si-SGLT1 groups were injected with $5 \mu \mathrm{l}$ of siRNA or $5 \mu \mathrm{l}$ of si-SGLT1 $(200 \mathrm{nmol} /$ $500 \mathrm{~g}$ ) in PBS, once a week. All rats were sacrificed after 16 weeks of feeding. The left ventricular tissues were removed and cut into pieces for histomorphological analysis.

\section{ELISA}

After the rats were fasted overnight, blood samples were obtained from the postcaval vein and processed for plasma extraction within $1 \mathrm{~h}$ (centrifuged at $3,000 \mathrm{xg}$ for $10 \mathrm{~min}$ at $4^{\circ} \mathrm{C}$ ), and the plasma was stored at $-80^{\circ} \mathrm{C}$ in polypropylene tubes for further analysis. The expression levels of collagen I, collagen III, and transforming growth factor- $\beta 1$ (TGF- $\beta 1$ ) in CFs and rat serum were detected using the rat collagen I Type I ELISA Kit (abx052369, Abbexa, United Kingdom), rat collagen type III ELISA Kit (abx573727, Abbexa), and TGF- $\beta 1$ ELISA Kit (PT878, Beyotime, Jiangsu, China), respectively, following the instructions of the manufacturer.

\section{Histology}

Tissues from rats were fixed using $10 \%$ buffered formalin, dehydrated, embedded in paraffin, and sectioned into $5 \mu \mathrm{m}$-thick sections. Hematoxylin and eosin (HE) staining was used to assess cardiac injury, whereas Masson's trichrome staining was used to detect collagen fibers, and the slides were observed under an optical microscope. For immunohistochemistry, sections were stained with a primary antibody against SGLT1 (1:200, Abcam) and then stained with a secondary antibody. After washing with PBS, the slides were incubated with 3,3'-diaminobenzidine. The detailed procedure has been described previously (Lin et al., 2019). Semiquantitative analysis was performed using image analysis software (Image-Pro Plus, Media Cybernetics).

\section{Wheat Germ Agglutinin Staining}

Slides were stained with Alexa Fluor 488-conjugated wheat germ agglutinin WGA (Sigma). In brief, slides were dewaxed, rehydrated, and blocked with 3\% BSA for $20 \mathrm{~min}$. The slides were then incubated in WGA solubilized in PBS for $30 \mathrm{~min}$ at room temperature in the dark. After washing with PBS, the sections were stained with DAPI (Invitrogen) for $5 \mathrm{~min}$ and images were acquired using a Nikon Eclipse Ti-U fluorescence microscope (Minato-ku, Tokyo, Japan). The cardiomyocyte size was determined by dividing the total area by the number of cardiomyocytes using Image J software (NIH, Bethesda, MD, Unites States).

\section{Statistical Analysis}

The experimental data are expressed as the mean \pm standard deviation. All statistical analyses were performed using GraphPad Prism 8.0 software (GraphPad Software, San Diego, CA, United States). Data are presented as the mean \pm standard deviation. The $t$-test was used to perform comparisons between the two different groups. One-way analysis of variance was used to compare multiple groups. Statistical significance was set at $p<0.05$.

\section{RESULTS}

\section{SGLT1 Is Upregulated in Diabetic Cardiac Tissues and High-Glucose-Induced Cardiac Fibroblasts}

The results of IPGTT revealed that blood glucose levels peaked half an hour after intraperitoneal injection, slowly decreased thereafter, and remained at $6-8 \mathrm{mmol} / \mathrm{L}$ in the control group 


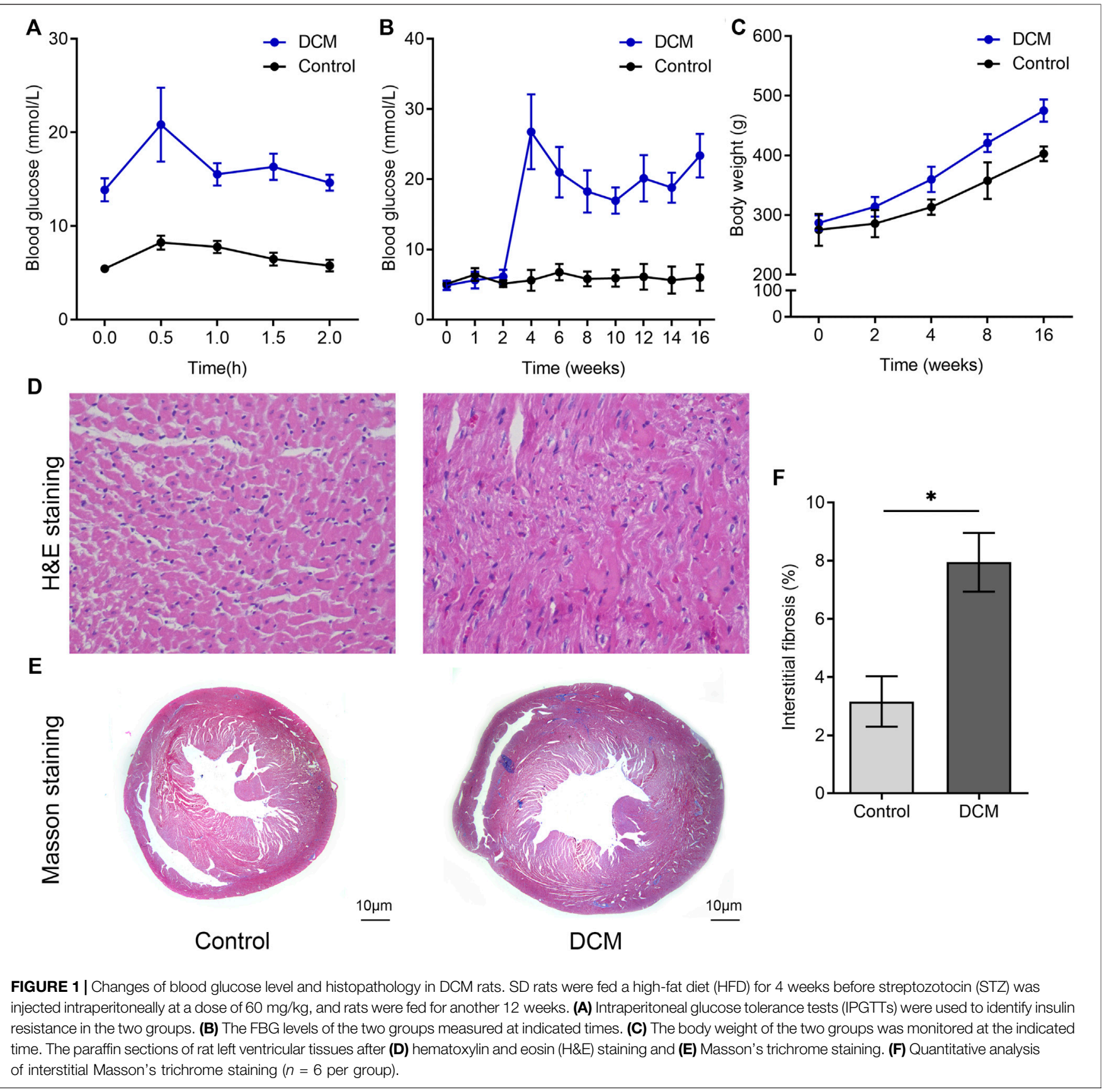

throughout the entire process. In contrast, the blood glucose levels of diabetic rats demonstrated evident hyperglycemia throughout the entire process (Figure 1A). Figure 1B shows that rats in the control group had a relatively stable FBG level, whereas the FBG level of the DCM group increased significantly after injection with STZ in the fourth week $(p<0.05)$. The body weight of the DCM group was higher than that of the control group (Figure 1C). HE and Masson staining were performed to examine the changes in cardiac pathology (Figures 1D,E). Increased interstitial fibrotic areas were observed in the DCM group compared to the NC group (Figure 1F).
We then validated that SGLT1 mRNA and protein levels were both upregulated in the DCM group compared to those in the control group (Figures 2A,B). Moreover, using immunohistochemistry, we found that $\mathrm{CFs}$ in diabetic rats expressed higher SGLT1 levels than those in normal rats (Figures 2C,D). In contrast to the control group, diabetic rats showed a significant increase in fibrosis-related proteins, including collagen I and collagen III expression (Figures 2C,E). In vitro, CFs changed from a long and thin shape to fusiform under HG conditions (Figure 2F), and the results of immunofluorescence revealed that the fluorescence intensity of SGLT1 was markedly increased in the HG medium (Figure 2G). 

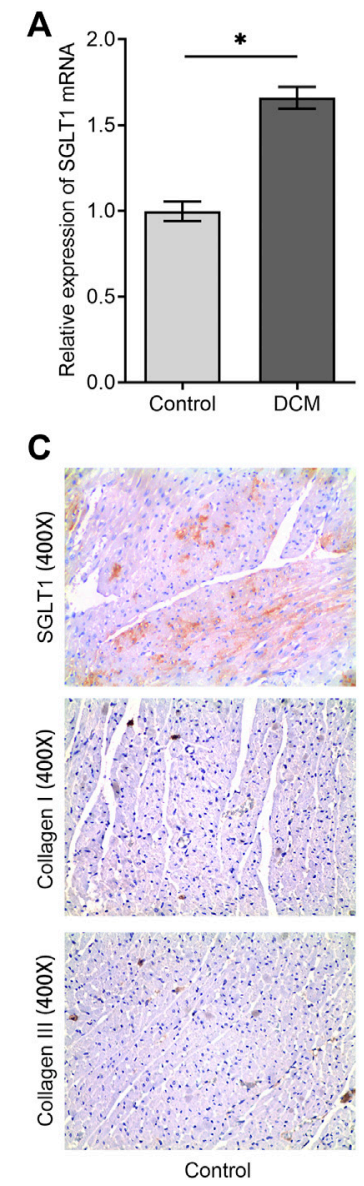

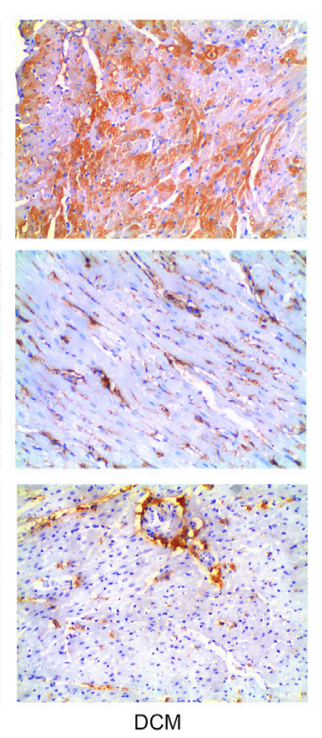

B


$\mathbf{F}$



$\mathbf{G}$

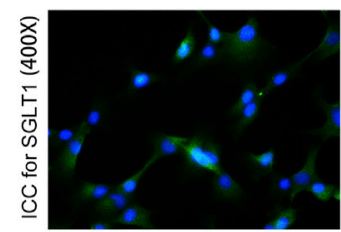

Control

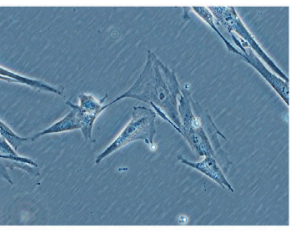

HG

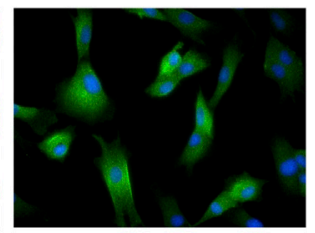

HG

FIGURE 2 | SGLT1 is downregulated in the experimental diabetic cardiac fibrosis model and high-glucose-induced CFs. (A) The mRNA level of SGLT1 was detected using RT-qPCR $(n=6)$. (B) The protein level of SGLT1 was analyzed using western blot analysis $(n=6)$. (C) The protein levels of SGLT1, collagen I, and collagen III were evaluated using immunohistochemical staining. Relative expression of SGLT1 (D) and collagen I and collagen III (E) was found using semiquantitative analysis based on immunohistochemistry. (F) The morphology of CFs with or without high-glucose stimulation was imaged under an optical microscope. (G) The protein level of SGLT1 was analyzed by immunofluorescent staining in high-glucose-induced CFs. (H) The protein levels of SGLT1, collagen I, and collagen III were analyzed using western blot analysis in high-glucose-induced CFs $(n=3)$.

Accordingly, western blotting analysis revealed that compared to the control group, the levels of SGLT1, collagen I, and collagen III proteins were significantly upregulated in the HG group (Figure $\mathbf{2 H}$ ).

\section{Knockdown of SGLT1 Inhibits High-Glucose-Induced Cardiac Fibroblast Activation}

To investigate the role of SGLT1 in CF activation, we knocked down SGLT1 by transfecting CFs with specific siRNAs against SGLT1. The results of RT-qPCR (Figure 3A) and western blotting (Figure 3B) analysis validated that SGLT1 si-RNA2 exerted the highest knockdown efficiency and was, therefore, chosen to perform the subsequent assays. Subsequent characterization of the CFs showed that HG stimulation significantly increased cell viability (Figure 3C) and migration (Figures 3D,E). We found that CFs with SGLT1 inhibition had markedly reduced cell viability and migration compared with those in the $\mathrm{HG}+$ si-NC group (Figures 3C-E). Furthermore, ELISA revealed that HG stimulation caused high expression of TGF- $\beta 1$, collagen I, and collagen III, indicating the synthesis of collagen in CFs, whereas the inhibition of SGLT1 effectively reversed this increase (Figures 3F-H). We further analyzed the potential involvement of the p38 mitogen-activated protein kinase (MAPK) and ERK1/2 signaling pathways in the regulatory role of SGLT1 in HG-mediated CF activation. As expected, HG significantly activated the phosphorylation of $\mathrm{p} 38$ MAPK and ERK1/2, whereas SGLT1 silencing reduced the effects of HG (Figure 3I). Therefore, these data indicate that SGLT1 regulates the function of CFs.

\section{Sodium-Glucose Cotransporter 1 Regulates p38 MAPK and ERK1/2 Signaling and Collagen Synthesis in Cardiac \\ Fibroblasts}

We further investigated the potential mechanism underlying the involvement of SGLT1 in the activation of CFs. SGLT1 overexpression was achieved by transfecting CFs with a 


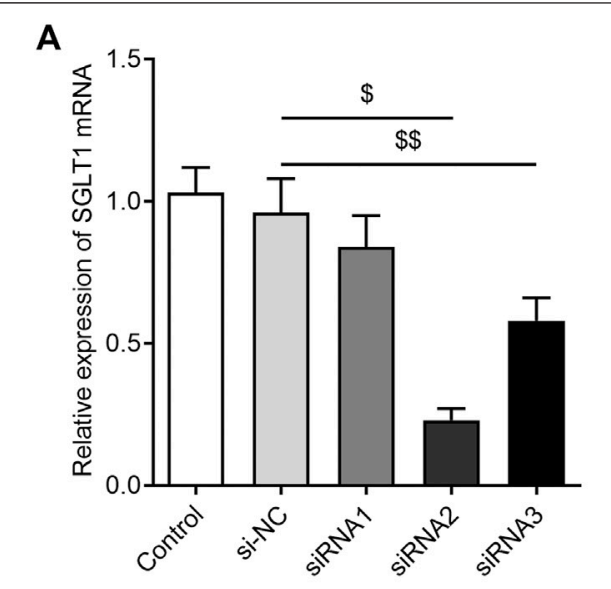

D
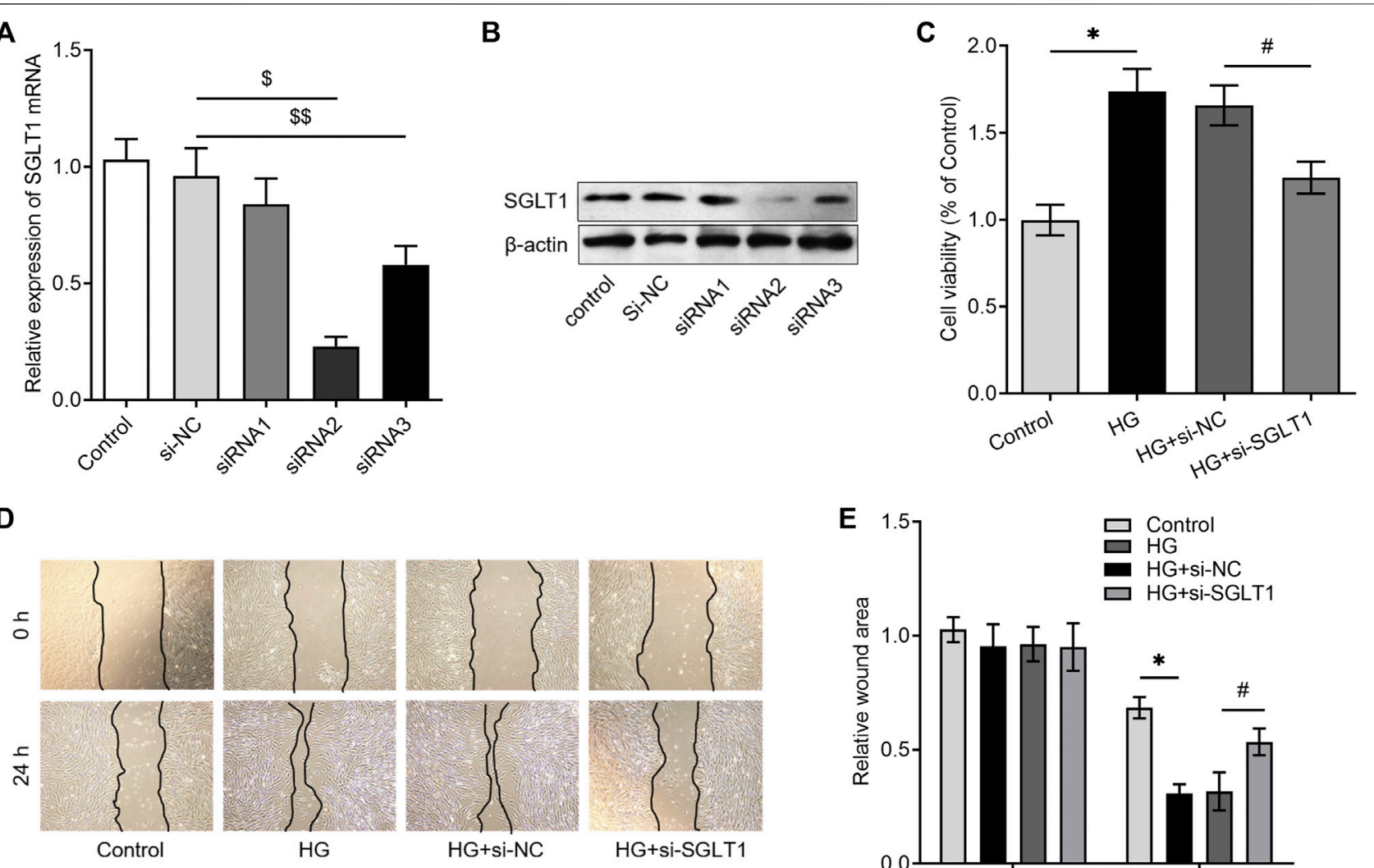

$\mathbf{F}$

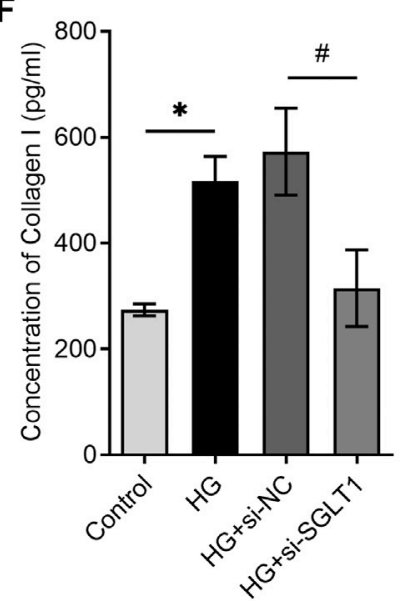

$\mathbf{G}$
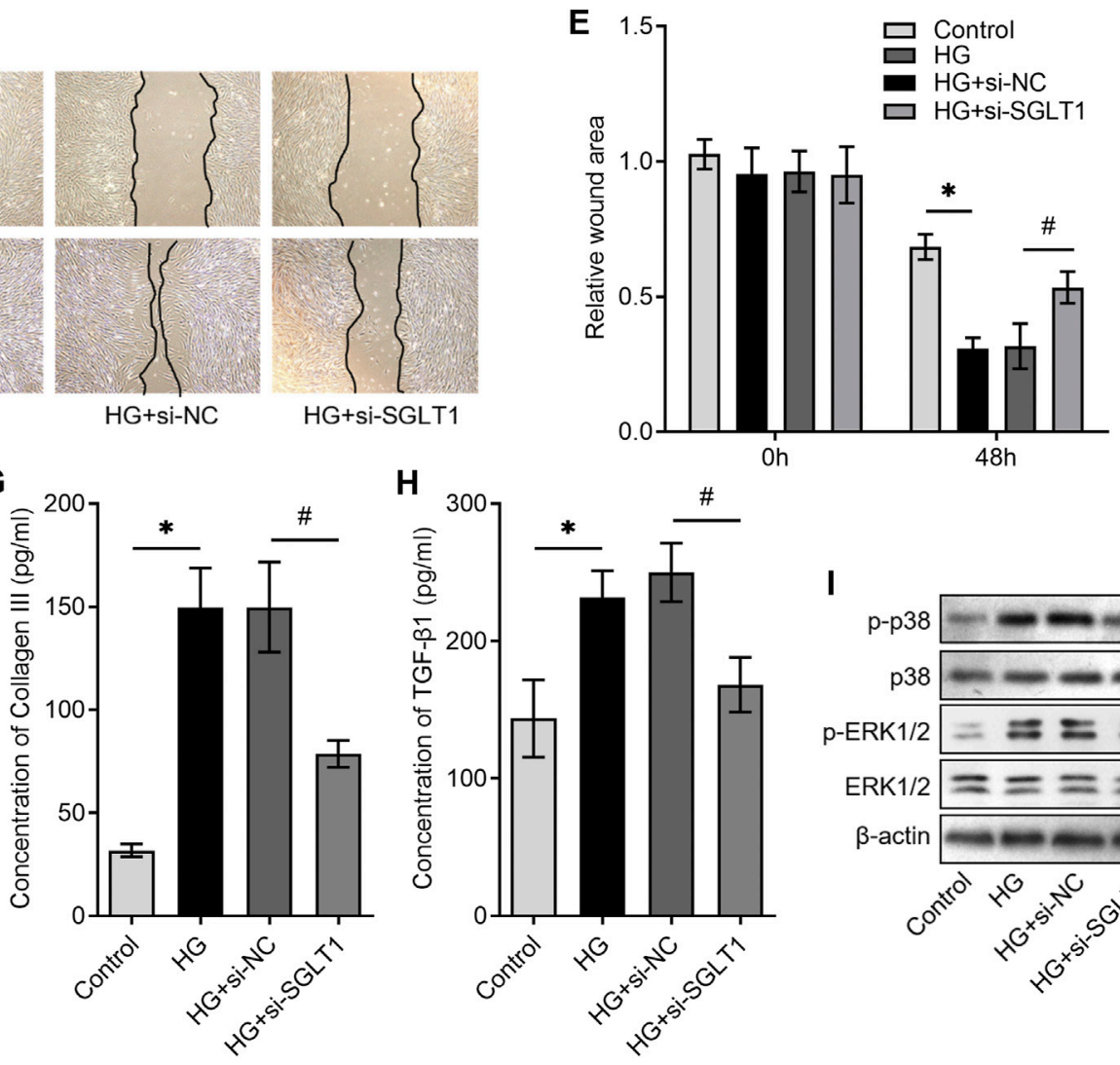

FIGURE 3 | Knockdown of SGLT1 inhibited high-glucose-induced CF activation. CFs were transfected with SGLT1 siRNAs, and SGLT1 mRNA and protein levels were detected using RT-qPCR (A) and western blotting, respectively (B). CCK-8 assay was used to detect the proliferation of CFs under high-glucose condition with or without SGLT1 inhibition. (D, E) The representative images of the wound-healing assay were obtained at 0 and $24 \mathrm{~h}$ after knockdown of SGLT1, and the migrative ability of CFs was compared. (F-H) ELISA was used to detect the levels of collagen-synthesis-related markers, including TGF- $\beta 1$, collagen I, and collagen III in the cell supernatant $(n=6)$. (I) Western blotting analysis was performed to investigate the phosphorylation levels of p38 mitogen-activated protein kinase (MAPK) and extracellular signal-regulated kinase (ERK)1/2 in CFs under high-glucose condition with or without SGLT1 inhibition.

plasmid containing the SGLT1 gene, and RT-qPCR and western blotting analysis revealed significantly higher SGLT1 levels in the SGLT1-transfected group when compared with the control group (Figures 4A,B). Interestingly, we found that the overexpression of SGLT1 significantly increased the proliferation of CFs under both normal and HG conditions (Figure 4C). In addition, the overexpression of SGLT1 effectively promoted the secretion of TGF- $\beta 1$, collagen I, and collagen III in the cell supernatant, under 

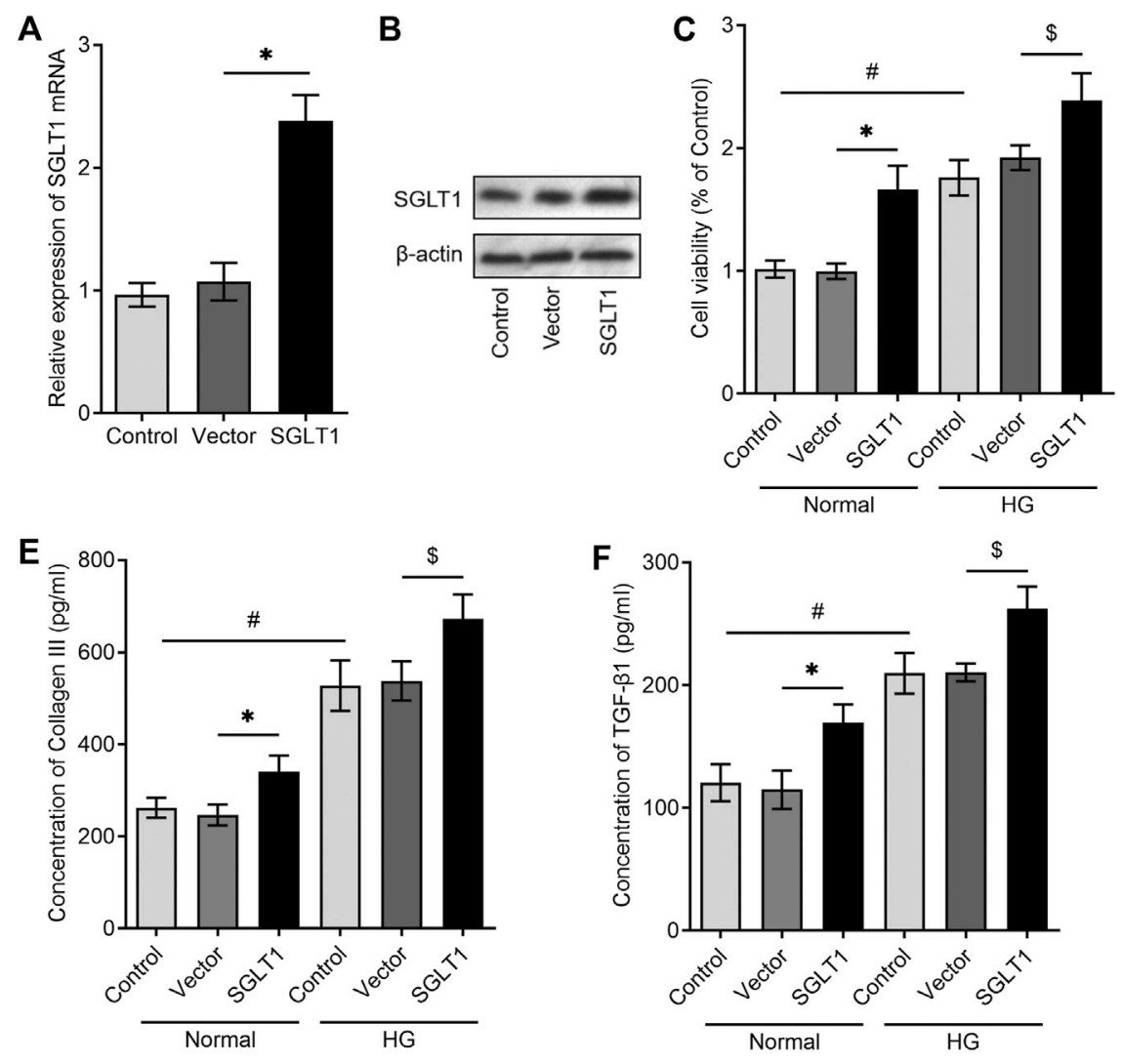



$\mathbf{G}$

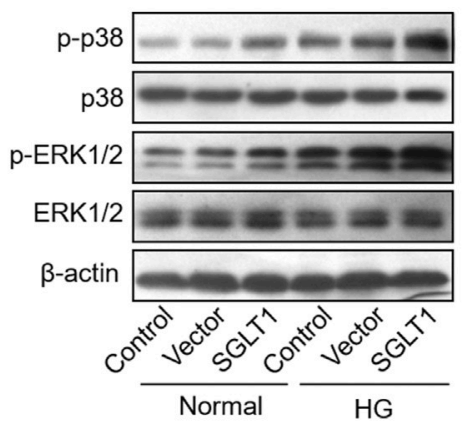

FIGURE 4 | SGLT1 overexpression promotes collagen release via the p38 and ERK1/2 signaling pathway in CFs. CFs were transfected with an SGLT1 plasmid or vector, and SGLT1 expression levels were detected using RT-qPCR (A) or western blotting analysis (B). (C) After overexpression of SGLT1 in CFs, CCK-8 assay was used to detect the proliferation of CFs under normal or high-glucose condition $(n=6)$. (D-F) After overexpressing SGLT1 in CFs, the collagen-synthesis-related markers, including TGF- $\beta 1$, collagen I, and collagen III, in the cell supernatant were measured using ELISA $(n=6)$. (G) After overexpressing SGLT1 in CFs, the protein levels of phosphorylated p38 and p-ERK1/2 were analyzed using western blotting in CFs both under normal or high-glucose condition $(n=3)$.

both normal and HG conditions (Figures 4D-F). Furthermore, overexpression of SGLT1 significantly increased p38 and p-ERK1/2 levels under both normal and HG conditions (Figure 4G). These data suggest that SGLT1 might activate CFs by activating the $\mathrm{p} 38$ and $\mathrm{p}$-ERK1/2 pathways.

\section{Sodium-Glucose Cotransporter 1 Inhibition Alleviates Fibrosis in the Diabetic Heart}

Since cardiac fibrosis and hypertrophy are important pathological structural features of DCM, we investigated whether SGLT1 regulates fibrosis and hypertrophy in diabetic hearts. We knocked down SGLT1 in rats with DCM by continuously administering si-SGLT1 via intravenous injection in the tail vein. As shown in Figures $\mathbf{5 A , B}$, the expression of SGLT1 mRNA and protein in the heart was reduced in the DCM + si-SGLT1 group when compared with that in the DCM + si-NC group. Accordingly, the immunohistochemistry results indicated that CFs expressed low SGLT1 levels after SGLT1 inhibition (Figure 5C). Interestingly, compared with the DCM + si-NC group, a significant reduction in interstitial fibrosis was observed in the DCM + si-SGLT1 group (Figure 5D). The hallmarks of fibrosis and protein levels of collagen I and collagen III were reduced by SGLT1 knockdown (Figures 5E,F). However, SGLT1 knockdown had no significant effect on cardiac hypertrophy, as examined by WGA staining (Figure 5G). Collectively, our results indicated that the knockdown of SGLT1 reduced cardiac fibrosis but had no effect on cardiac hypertrophy in DCM.

\section{DISCUSSION}

Increasing attention has been placed on the utility of SGLT2 inhibitors because, in addition to controlling blood glucose levels, they have been shown to provide significant cardiovascular benefits in T2DM patients (Packer et al., 2021). Rather than SGLT2, recent findings have also emphasized the potential role of SGLT1 in the development of cardiovascular diseases. The myocardial expression of SGLT1 in humans is altered in various cardiovascular disease states. Compared with controls, left ventricular SGLT1 mRNA and protein expression was significantly upregulated in heart failure patients with DCM (Sayour et al., 2020). Individuals carrying loss-of-function mutations in the SGLT1 gene are estimated to have a lower 


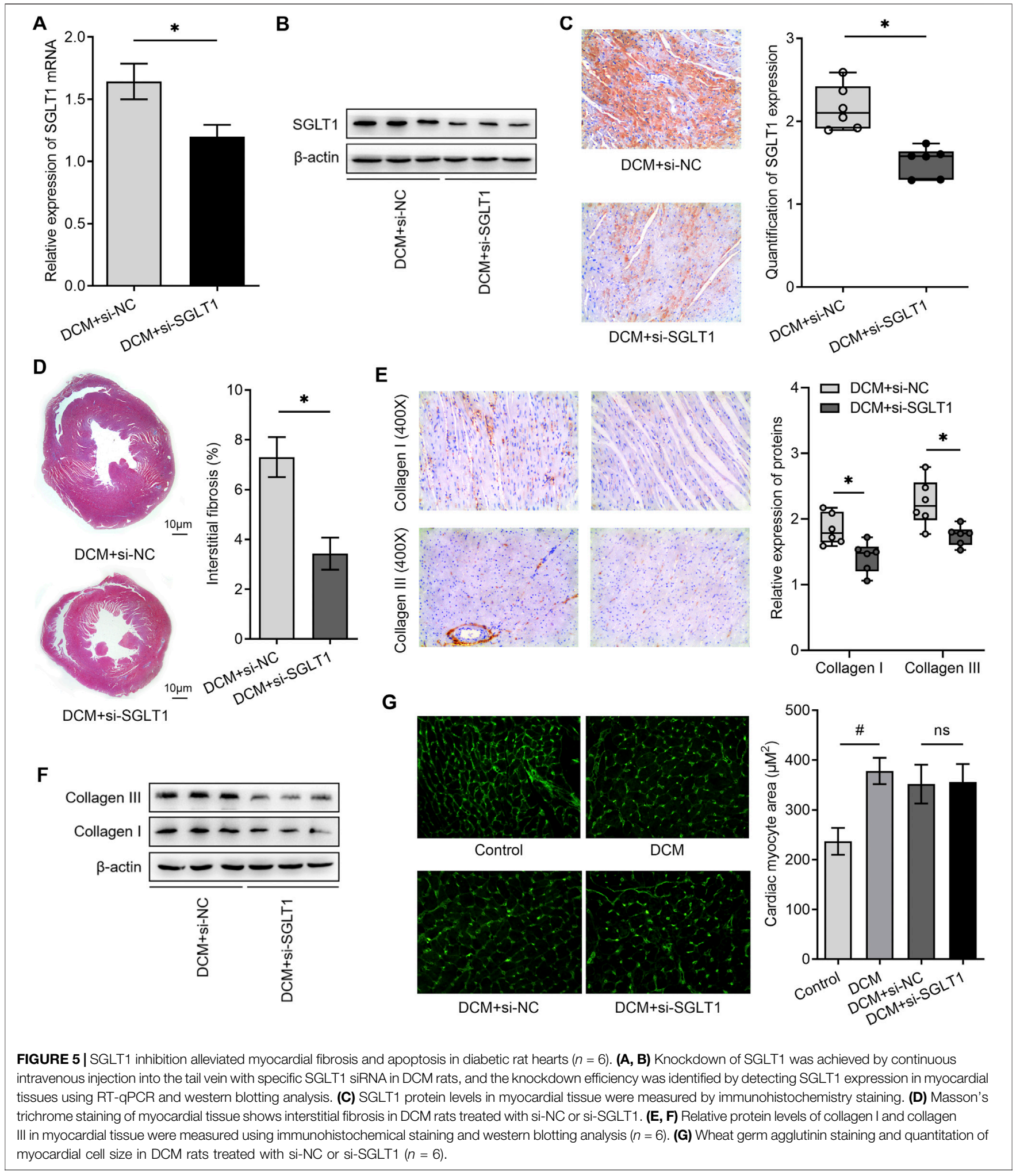

risk of developing heart failure, driven by mitigation of postprandial hyperglycemic episodes (Seidelmann et al., 2018). In endothelial cells, angiotensin II upregulates SGLT1 expression to promote sustained oxidative stress, and inhibition of SGLT1 appears to be an attractive strategy to enhance protective endothelial function (Park et al., 2021). Apart from cardiomyocytes and endothelial cells in the heart, we first identified that SGLT1 was expressed in human CFs (Meng 
et al., 2018), and in the present study, the significant finding was that an increase in SGLT1 expression in rat hearts triggered the development of cardiac fibrosis through activation of CFs by upregulating the p38 MAPK and ERK1/2 signaling pathways.

Targeting SGLT1 has also been found to have cardioprotective effects in DCM. RNA-mediated inhibition of SGLT1 gene glycemic variability and cardiac damage were seen in type 2 diabetes mellitus mice in vivo (Sun et al., 2021). In cultured cardiomyocytes, SGLT1 knockdown restored cell proliferation, suppressed reactive oxygen species, and induced cytotoxicity (Chai et al., 2021). These data supported the notion that SGLT1 might serve as a target for myocardial injury in the diabetic heart. It is well known that $\mathrm{CF}$ activation plays an essential role during the development of cardiac fibrosis. However, the role of SGLT1 in $\mathrm{CF}$ activation remains unclear.

Several lines of experimental evidence suggest that SGLT1 silencing may attenuate cardiac fibrosis. Ramratnam et al. (2014) demonstrated that cardiac overexpression of SGLT1 increases collagen I gene expression and interstitial fibrosis in mouse hearts. Another study found that SGLT1 knockout downregulated CTGF and collagen I gene expression and interstitial fibrosis in pressure-overload-increased mouse hearts (Matsushita et al., 2018). Similar to these studies, we also found that knockdown of SGLT in diabetic hearts suppressed the synthesis of TGF- $\beta 1$, collagen I, and collagen III. Furthermore, in cultured CFs, we found that SGLT1 regulates cell proliferation and collagen synthesis, suggesting the role of SGLT1 in regulating CF activation. To the best of our knowledge, this study is the first to demonstrate that SGLT1 regulates the activation of CFs in DCM.

Our in vitro experiments showed that $\mathrm{HG}$ upregulated SGLT1 expression in CFs, which was accompanied by an increase in the abundance of p-p38 and p-ERK1/2. SGLT1 overexpression significantly induced the abundance of these proteins in CFs under both normal and HG conditions. TGF- $\beta 1$ stimulation in CFs resulted in increased proliferation, increased collagen I and collagen III expression, and increased p38 and ERK1/2 phosphorylation (Xu et al., 2017), whereas inhibition of the activation of p38 kinase and ERK1/2 could effectively attenuate cardiac fibrosis (Tao et al., 2016). Activation of MAPKs participates in the upregulation of cerebral SGLT-1 expression (Yamazaki et al., 2018). Moreover, the relationship between the SGLT1 and MAPK signaling pathways in the heart has also been reported in our previous study (Lin et al., 2021). Based on the abovementioned results, we deduced that the increase in SGLT1 expression in the diabetic heart is involved in triggering CF proliferation and subsequent cardiac fibrosis.

Furthermore, we noticed that the study performed by Matsushita et al. suggested that SGLT1 knockout could prevent chronic pressure-overload-induced hypertrophic cardiomyopathy (Matsushita et al., 2018). However, in our study, we found that knockdown of SGLT1 had no effect on hyperglycemia-related hypertrophy in diabetic hearts. This discrepancy may be because of the differences in experimental animal models. We used SD rats to establish a DCM model, and Matsushita et al. (2018) used mice that underwent transverse aortic constriction surgery. The other significant difference is that we only knocked down SGLT1 in rats using specific siRNA, rather than using gene knockout technology. A previous study suggested that SGLT1-deficient mice need to consume a glucose-galactosefree diet because they show symptoms of glucose-galactose malabsorption syndrome (Gorboulev et al., 2012). Therefore, SGLT1 knockout may not be appropriate in DCM. More studies are needed to investigate the exact role of SGLT1 in cardiac hypertrophy.

In summary, our study evaluated the changes in the expression of SGLT1 in the progression of diabetic cardiac fibrosis and identified a significant increase in SGLT1 expression in the diabetic heart. SGLT1 is involved in cardiac fibrosis via the p38 and ERK1/2 signaling pathways. Our findings suggest that SGLT1 is a potential therapeutic target for the prevention of diabetic cardiac fibrosis.

\section{DATA AVAILABILITY STATEMENT}

The raw data supporting the conclusions of this article will be made available by the authors, without undue reservation.

\section{ETHICS STATEMENT}

The animal study was reviewed and approved by the Medicine Animal Welfare Committee of Shaoxing People's Hospital.

\section{AUTHOR CONTRIBUTIONS}

LM and HU designed the project; HL and LG performed animal experiments and analyzed the data; LM and HL performed in vitro experiments and wrote the draft manuscript; HG supervised and funded the project; and $\mathrm{HU}$ and $\mathrm{HG}$ made the modification.

\section{FUNDING}

This research was supported by grants from the Zhejiang Provincial Natural Science Foundation of China (No. LGF19H020006 and LGF21H020001), Health Science and Technology Plan Innovative Talents Project of Zhejiang Province (No. 2021RC032), the National Natural Science Foundation of China (82000252), Science and Technology Project of Shaoxing (No. 2019B24004) and the Key Laboratory of Cardio-cerebral Vascular Disease Rehabilitation Technology Innovation and Application of Shaoxing City (2020ZDSYS02). 


\section{REFERENCES}

Bugger, H., and Abel, E. D. (2014). Molecular Mechanisms of Diabetic Cardiomyopathy. Diabetologia 57 (4), 660-671. doi:10.1007/s00125-014-3171-6

Chai, Q., Miao, J., Liu, M., Zhang, Z., Meng, Z., and Wu, W. (2021). Knockdown of SGLT1 Prevents the Apoptosis of Cardiomyocytes Induced by Glucose Fluctuation Via Relieving Oxidative Stress and Mitochondrial Dysfunction. Biochem. Cell Biol. 99 (3), 356-363. doi:10.1139/bcb-2020-0491

Feng, Y., Xu, W., Zhang, W., Wang, W., Liu, T., and Zhou, X. (2019). LncRNA DCRF Regulates Cardiomyocyte Autophagy by Targeting miR-551b-5p in Diabetic Cardiomyopathy. Theranostics 9 (15), 4558-4566. doi:10.7150/thno.31052

Frangogiannis, N. G. (2021). Cardiac Fibrosis. Cardiovasc Res. 117 (6), 1450-1488. doi:10.1093/cvr/cvaa324

Gorboulev, V., Schürmann, A., Vallon, V., Kipp, H., Jaschke, A., Klessen, D., et al. (2012). Na+-D-glucose Cotransporter SGLT1 Is Pivotal for Intestinal Glucose Absorption and Glucose-dependent Incretin Secretion. Diabetes 61 (1), 187-196. doi:10.2337/db11-1029

Gyimesi, G., Pujol-Giménez, J., Kanai, Y., and Hediger, M. A. (2020). Sodiumcoupled Glucose Transport, the SLC5 Family, and Therapeutically Relevant Inhibitors: from Molecular Discovery to Clinical Application. Pflugers Arch. Eur. J. Physiol. 472 (9), 1177-1206. doi:10.1007/s00424-020-02433-x

Hirose, M., Matsushita, N., Ishida, N., Ibi, M., and Saito, M. (2018). Roles of Sodium-Glucose Cotransporter 1 (SGLT1) in the Induction of Cardiac Remodeling. Yakugaku Zasshi 138 (7), 939-943. doi:10.1248/yakushi.1700223-2

Lin, H., Zhang, J., Ni, T., Lin, N., Meng, L., Gao, F., et al. (2019). Yellow Wine Polyphenolic Compounds Prevents Doxorubicin-induced Cardiotoxicity through Activation of the Nrf2 Signalling Pathway. J. Cel Mol Med 23 (9), 6034-6047. doi:10.1111/jcmm.14466

Lin, N., Lin, H., Yang, Q., Lu, W., Sun, Z., Sun, S., et al. (2021). SGLT1 Inhibition Attenuates Apoptosis in Diabetic Cardiomyopathy via the JNK and P38 Pathway. Front. Pharmacol. 11, 598353. doi:10.3389/fphar.2020.598353

Matsushita, N., Ishida, N., Ibi, M., Saito, M., Sanbe, A., Shimojo, H., et al. (2018). Chronic Pressure Overload Induces Cardiac Hypertrophy and Fibrosis via Increases in SGLT1 and IL-18 Gene Expression in Mice. Int. Heart J. 59 (5), 1123-1133. doi:10.1536/ihj.17-565

Meng, L., Uzui, H., Guo, H., and Tada, H. (2018). Role of SGLT1 in High Glucose Level-Induced MMP-2 Expression in Human Cardiac Fibroblasts. Mol. Med. Rep. 17 (5), 6887-6892. doi:10.3892/mmr.2018.8688

Packer, M., Anker, S. D., Butler, J., Filippatos, G., Ferreira, J. P., Pocock, S. J., et al. (2021). Empagliflozin in Patients with Heart Failure, Reduced Ejection Fraction, and Volume Overload. J. Am. Coll. Cardiol. 77 (11), 1381-1392. doi:10.1016/j.jacc.2021.01.033

Park, S. H., Belcastro, E., Hasan, H., Matsushita, K., Marchandot, B., Abbas, M., et al. (2021). Angiotensin II-Induced Upregulation of SGLT1 and 2 Contributes to Human Microparticle-Stimulated Endothelial Senescence and Dysfunction: Protective Effect of Gliflozins. Cardiovasc. Diabetol. 20 (1), 65. doi:10.1186/ s12933-021-01252-3

Ramratnam, M., Sharma, R. K., D’Auria, S., Lee, S. J., Wang, D., Huang, X. Y., et al. (2014). Transgenic Knockdown of Cardiac Sodium/glucose Cotransporter 1 (SGLT1) Attenuates PRKAG2 Cardiomyopathy, whereas Transgenic Overexpression of Cardiac SGLT1 Causes Pathologic Hypertrophy and Dysfunction in Mice. J. Am. Heart Assoc. 3 (4), e000899. doi:10.1161/jaha.114.000899

Sano, R., Shinozaki, Y., and Ohta, T.. Sodium-glucose Co-transporters: Functional Properties and Pharmaceutical Potential. J. Diabetes Investig., 2020.11(4): 770-782. doi: doi:10.1111/jdi.13255
Sawa, Y., Saito, M., Ishida, N., Ibi, M., Matsushita, N., Morino, Y., et al. (2020) Pretreatment with KGA-2727, a Selective SGLT1 Inhibitor, Is Protective against Myocardial Infarction-Induced Ventricular Remodeling and Heart Failure in Mice. J. Pharmacol. Sci. 142 (1), 16-25. doi:10.1016/ j.jphs.2019.11.001

Sayour, A. A., Oláh, A., Ruppert, M., Barta, B. A., Horváth, E. M., Benke, K., et al. (2020). Characterization of Left Ventricular Myocardial Sodium-Glucose Cotransporter 1 Expression in Patients with End-Stage Heart Failure. Cardiovasc. Diabetol. 19 (1), 159. doi:10.1186/s12933-020-01141-1

Seferovic, P. M., and Paulus, W. J. (2015). Clinical Diabetic Cardiomyopathy: a Two-Faced Disease with Restrictive and Dilated Phenotypes. Eur. Heart J. 36 (27), 1718-1727. 1727a-1727c. doi:10.1093/eurheartj/ehv134

Seidelmann, S. B., Feofanova, E., Yu, B., Franceschini, N., Claggett, B., Kuokkanen, M., et al. (2018). Genetic Variants in SGLT1, Glucose Tolerance, and Cardiometabolic Risk. J. Am. Coll. Cardiol. 72 (15), 1763-1773. doi:10.1016/ j.jacc.2018.07.061

Song, P., Onishi, A., Koepsell, H., and Vallon, V. (2016). Sodium Glucose Cotransporter SGLT1 as a Therapeutic Target in Diabetes Mellitus. Expert Opin. Ther. Targets 20 (9), 1109-1125. doi:10.1517/14728222.2016.1168808

Sun, Z., Chai, Q., Zhang, Z., Lu, D., Meng, Z., and Wu, W. (2021). Inhibition of SGLT1 Protects against Glycemic Variability-Induced Cardiac Damage and Pyroptosis of Cardiomyocytes in Diabetic Mice. Life Sci. 271, 119116. doi:10.1016/j.lfs.2021.119116

Tao, L., Bei, Y., Chen, P., Lei, Z., Fu, S., Zhang, H., et al. (2016). Crucial Role of miR433 in Regulating Cardiac Fibrosis. Theranostics 6 (12), 2068-2083. doi:10.7150/thno.15007

Wang, L., Cai, Y., Jian, L., Cheung, C. W., Zhang, L., and Xia, Z. (2021). Impact of Peroxisome Proliferator-Activated Receptor- $\alpha$ on Diabetic Cardiomyopathy. Cardiovasc. Diabetol. 20 (1), 2. doi:10.1186/s12933-020-01188-0

Wood, I. S., and Trayhurn, P. (2003). Glucose Transporters (GLUT and SGLT): Expanded Families of Sugar Transport Proteins. Br. J. Nutr. 89 (1), 3-9. doi:10.1079/bjn2002763

Xu, Y., Xiao, H., Luo, H., Chen, Y., Zhang, Y., Tao, L., et al. (2017). Inhibitory Effects of Oxymatrine on TGF-B1-Induced Proliferation and Abnormal Differentiation in Rat Cardiac Fibroblasts via the p38MAPK and ERK1/2 Signaling Pathways. Mol. Med. Rep. 16 (4), 5354-5362. doi:10.3892/ mmr.2017.7277

Yamazaki, Y., Arita, K., Harada, S., and Tokuyama, S. (2018). Activation of C-Jun N-Terminal Kinase and P38 after Cerebral Ischemia Upregulates Cerebral Sodium-Glucose Transporter Type 1. J. Pharmacol. Sci. 138 (4), 240-246. doi:10.1016/j.jphs.2017.02.016

Zhang, J., Zhang, M., Yang, Z., Huang, S., Wu, X., Cao, L., et al. (2020). PDCD4 Deficiency Ameliorates Left Ventricular Remodeling and Insulin Resistance in a Rat Model of Type 2 Diabetic Cardiomyopathy. BMJ Open Diabetes Res. Care 8 (1), e001081. doi:10.1136/bmjdrc-2019-001081

Conflict of Interest: The authors declare that the research was conducted in the absence of any commercial or financial relationships that could be construed as a potential conflict of interest.

Copyright (C) 2021 Lin, Guan, Meng, Uzui and Guo. This is an open-access article distributed under the terms of the Creative Commons Attribution License (CC BY). The use, distribution or reproduction in other forums is permitted, provided the original author(s) and the copyright owner(s) are credited and that the original publication in this journal is cited, in accordance with accepted academic practice. No use, distribution or reproduction is permitted which does not comply with these terms. 